\title{
Query translation architecture for Malay-English cross-language information retrieval system
}

\begin{abstract}
This paper discusses research on query translation events in Malay-English Cross-Language Information Retrieval (CLIR) system. We assume that by improving query translation accuracy, we can improve the information retrieval performance. The dictionary-based CLIR system facing three main problems: translation ambiguity; compound and phrase handling and proper names translation. The use of natural language processing (NLP) techniques, such as stemming, Part-of-Speech (POS) tagging is useful in query translation process. Hence, ngram matching technique has successfully applied to information retrieval (IR) system for phrases and proper names translation. The proposed query translation architecture consist of stemming, Part-of-Speech (POS) tagging and n-gram matching techniques is useful in CLIR system as well as search engine application.
\end{abstract}

Keyword: Component; Cross-language information retrieval; N-gram matching; Part-ofSpeech (POS) tagging; Query ambiguity; Query translation 